\title{
Dynamical Scaling Implications of Ferrari, Prähofer, and Spohn's Remarkable Spatial Scaling Results for Facet-Edge Fluctuations
}

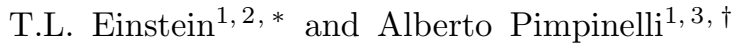 \\ ${ }^{1}$ Department of Physics, University of Maryland, College Park, Maryland 20742-4111, USA \\ ${ }^{2}$ Condensed Matter Theory Center, University of Maryland, College Park, Maryland 20742-4111, USA \\ ${ }^{3}$ Rice Quantum Institute, Rice University, Houston, Texas 77005, USA
}

(Dated: June 17, 2021)

\begin{abstract}
Spurred by theoretical predictions from Spohn and coworkers [Phys. Rev. E 69, 035102(R) (2004)], we rederived and extended their result heuristically as well as investigated the scaling properties of the associated Langevin equation in curved geometry with an asymmetric potential. With experimental colleagues we used STM line scans to corroborate their prediction that the fluctuations of the step bounding a facet exhibit scaling properties distinct from those of isolated steps or steps on vicinal surfaces. The correlation functions was shown to go as $t^{0.15(3)}$ decidedly different from the $t^{0.26(2)}$ behavior for fluctuations of isolated steps. From the exponents, we were able to categorize the universality, confirming the prediction that the non-linear term of the KPZ equation, long known to play a central role in non-equilibrium phenomena, can also arise from the curvature or potential-asymmetry contribution to the step free energy. We also considered, with modest Monte Carlo simulations, a toy model to show that confinement of a step by another nearby step can modify as predicted the scaling exponents of the step's fluctuations. This paper is an expansion of a celebratory talk at the $95^{\text {th }}$ Rutgers Statistical Mechanics Conference, May 2006.
\end{abstract}

\section{INTRODUCTION}

Fluctuations of steps on surfaces play a central role in determining their impact on surface processes and the evolution of surface morphology. In the past nearly-two decades, the step continuum model has allowed several successful quantitative correlations of direct observations of step fluctuations with kinetic and thermodynamic descriptions of nanoscale structural evolution [1 5], bridging from the atomistic and nanoscale to the mesoscale. For steps on flat or vicinal (misoriented modestly from a facet orientation) surfaces, there are two well-defined scaling behaviors for temporal correlations, corresponding to cases B and A, conserved and non-conserved dynamics, respectively, in the framework of dynamic critical phenomena [6]. Several examples of both behaviors have been observed experimentally in physical systems [1, 2, 17] and numerically in Monte Carlo simulations [8 10].

For complex structures where mass transport is limited by geometry, the fundamental question of how fluctuations behave in a constrained environment becomes experimentally accessible. These issues become particularly important for smaller structures, especially nanostructures, where issues of finite volume (shape effects and volume conservation) become non-negligible [11, 12]. Although the step can still be viewed as a 1D interface obeying a Langevin-type equation of motion, not only local deformation but global effects must be considered when calculating the step chemical potential. These considerations alter the equation of motion, including the noise term, resulting in different universality classes of dynamic scaling [13] (see Table II below).

\footnotetext{
* einstein@umd.edu

$\dagger$ ap19@rice.edu
}

Thus, it was especially enlightening and inspiring to read of a well-defined intermediate scaling regime in Ferrari, Prähofer, and Spohn's stimulating paper 14 (hereafter FPS) (as well as related works [15 17]), in which they computed the scaling of equilibrium fluctuations of an atomic ledge bordering a crystalline facet surrounded by rough regions of the equilibrium crystal shape in their examination of a 3D Ising corner (Fig. 1). We refer to this boundary edge as the "shoreline" since it is the edge of an island-like region-the crystal facet-surrounded by a "sea" of steps.

FPS derive an intriguing exact result, concerning how the width $w$ of shoreline fluctuations scales as a function of the linear size of the facet. This length corresponds to the length of a step or the linear dimension of an island (or its circumference). This length is often called $L$ [18, 19] and other times $\ell$ [20] (while $\ell$ has a closely related but slightly different meaning in Ref. [14]). To prevent any possible confusion, we denote this length by the Polish crossed $L, E$, in this paper, following the notation used in a ceremonial presentation on this subject [21. We anticipate that

$$
w \sim E^{\alpha}
$$

where the value of roughness exponent $\alpha$ depends on the mode of mass transport and the geometry of the step. For the step that serves as the border a two-dimensional (2D) island on a high-symmetry crystal plane, one expects (and finds in physical and numerical experiments) that $w \sim E^{1 / 2}$, i.e. $\alpha=1 / 2$, since this step performs a random walk 22 .

FPS show that, as we quipped in the title of our paper 20, "a crystal facet is not an island". Indeed, they find that instead of the expected random-walk behavior,

$$
w \sim E^{1 / 3}
$$


i.e. $\alpha=1 / 3$, for a crystal facet. They prove that the origin of the unusual $E^{1 / 3}$ scaling lies in the step-step interactions between the facet ledge and the neighboring steps under conditions of conserved volume. Note that this value of $\alpha$ is intermediate between $\alpha=1 / 2$ for isolated steps and $\alpha=0(w \sim \ln (L)))$ [23] for a step on a vicinal surface, i.e. in a step train.

FPS's formidable calculation is based on the use of free spinless fermions, transfer matrices, random-matrix properties, Airy functions, and specific models; as a purely static result, it does not address the question of the time behavior of step fluctuations, which are easier to measure experimentally.

This article is an expansion of a celebratory talk 21] which described the impact of FPS on our research, in particular the results found in three publications [19, 20, 24. In Section II we summarize highlights of FPS that motivated and underpinned our subsequent work. In Section III we describe the relevant correlation functions. Next we present a heuristic derivation extending the reasoning of Pimpinelli et al. [18] that leads to the dynamic scaling of shoreline fluctuations, as well as the static result of FPS. Then we present a more formal analysis of scaling for curved steps in an asymmetric potential. In Section IV we describe experiments using scanning tunneling microscopy (STM) that demonstrate the novel scaling behavior in a physical system. In Section V we present Monte Carlo results for a toy model that shows in a simple system the effect of a neighboring step on the fluctuations of a step. The Conclusion section offers some final remarks.

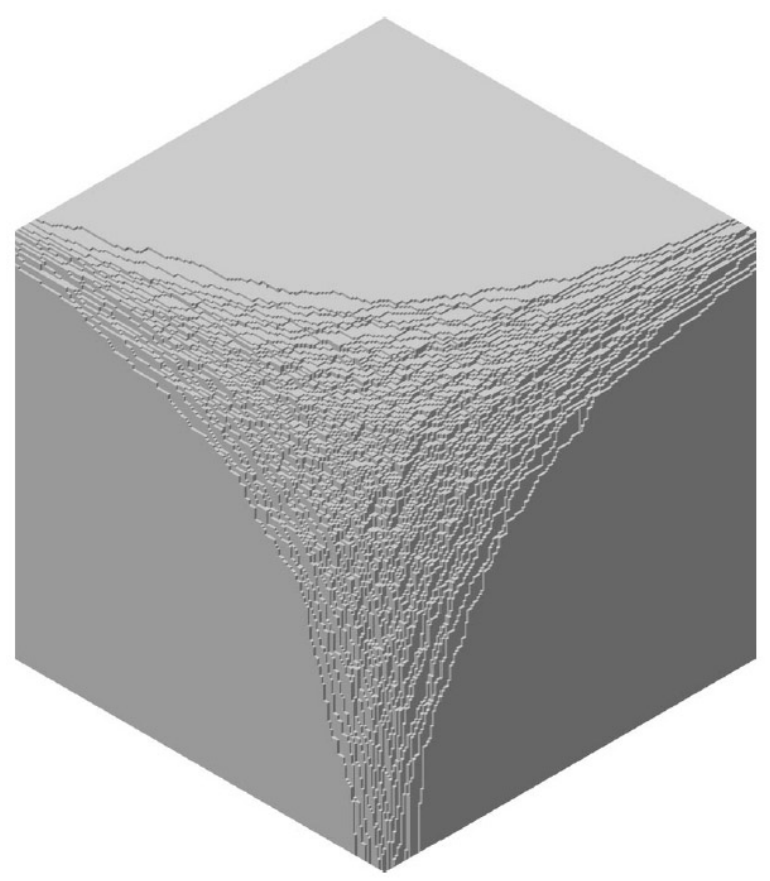

FIG. 1. Simple-cubic crystal corner viewed from the $\{111\}$ direction, from Refs. 14 17.
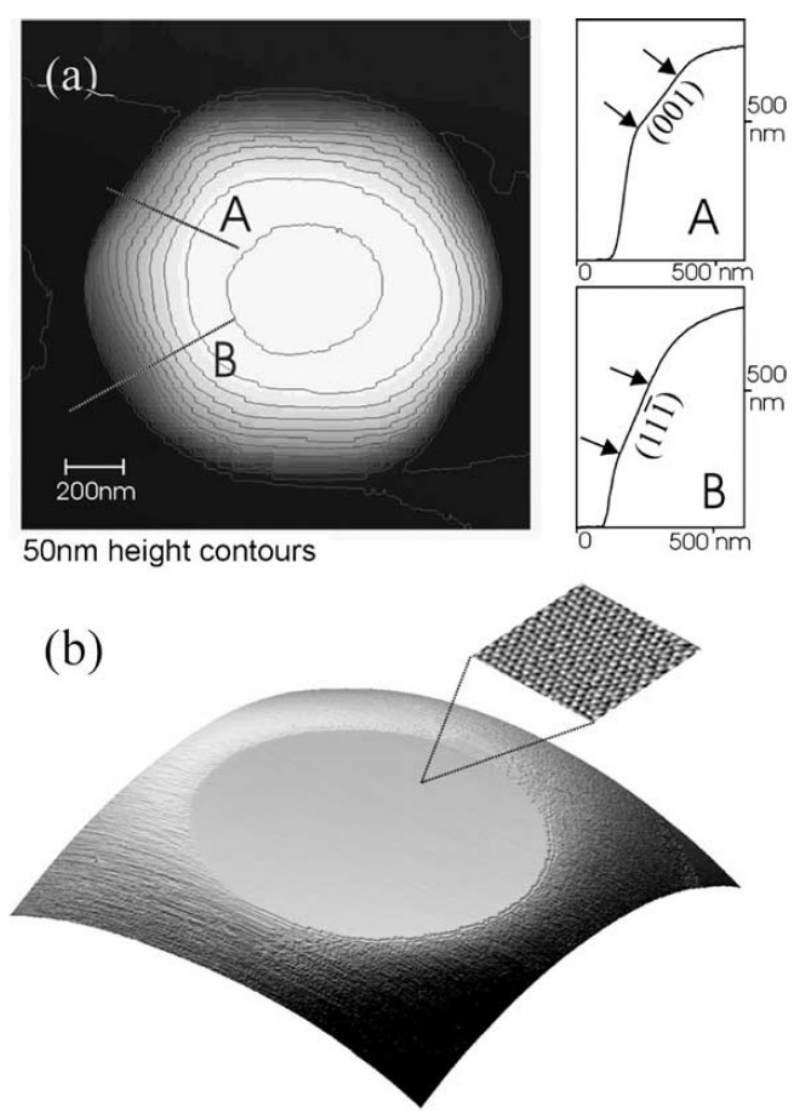

FIG. 2. (a) Micron-size lead crystal (supported on Ru) imaged with a variable-temperature STM at $T=95^{\circ} \mathrm{C}$. Annealing at $T=95^{\circ} \mathrm{C}$ for 20 hours allowed it to obtain its stable, regular shape. Lines marked $\mathrm{A}$ and $\mathrm{B}$ indicate location of profiles. Profile A crosses a $\left(\begin{array}{lll}0 & 0 & 1\end{array}\right)$-side facet, while profile B a (1 11 1)-side facet. (b) $770 \mathrm{~nm} \times 770 \mathrm{~nm}$ section of the top part of a Pb-crystal. The insert shows a $5.3 \mathrm{~nm} \times 5.3 \mathrm{~nm}$ area of the top facet, confirming its ( $\left.\begin{array}{lll}1 & 1 & 1\end{array}\right)$-orientation. Both the main image and the insert were obtained at $T=110^{\circ} \mathrm{C}$; from Ref. 25].

\section{SUMMARY OF HIGHLIGHTS OF FPS}

FPS assume that there are no interactions between steps beyond entropic (i.e., the steric repulsions arising from the fact that steps cannot cross), so that the step configurations can be mapped to the world lines of free spinless fermions; the entropic repulsion is captured by the fermionic Pauli condition [26. A key new feature compared to treatments of vicinal surfaces is that the volume of the crystallite is conserved. Their fermionic Hamiltonian $\mathcal{H}_{F}$ is

$$
\mathcal{H}_{F}=\sum_{j}\left(-a_{j}^{\dagger} a_{j+1}-a_{j+1}^{\dagger} a_{j}+2 a_{j}^{\dagger} a_{j}+\frac{j}{\lambda} a_{j}^{\dagger} a_{j}\right),
$$

where $\lambda^{-1}$ is a Lagrange multiplier associated with conserved volume. See Fig. 3. It is this final term that is 
new in their treatment. Its asymmetry is key to the novel behavior they find. They then derive an exact result for the step density in terms of $J_{j}$, the Bessel function of integer order $j$, and its derivative. Near the shoreline they find

$$
\lim _{\lambda \rightarrow \infty} \lambda^{1 / 3} \rho_{\lambda}\left(\lambda^{1 / 3} x\right)=-x(\operatorname{Ai}(x))^{2}+\left(\operatorname{Ai}^{\prime}(x)\right)^{2},
$$

where $\rho_{\lambda}$ is the step density (for the particular value of $\lambda)$.

The presence of the Airy function Ai results from the asymmetric potential implicit in $\mathcal{H}_{F}$ and preordains exponents involving $1 / 3$. The variance of the wandering of the shoreline, the top fermionic world line in Fig. 3 and denoted by $b$, is given by

$$
\operatorname{Var}\left[b_{\lambda}(t)-b_{\lambda}(0)\right] \cong \lambda^{2 / 3} g\left(\lambda^{-2 / 3} t\right)
$$

where $t$ is the fermionic "time" along the step; $g(s) \sim$ $2|s|$ for small $s$ (diffusive meandering) and $\sim 1.6264-$ $2 / s^{2}$ for large $s$. They then set $\lambda$ to a scaling parameter $\ell=(4 N / 1.202 \ldots)^{1 / 3}$, where $1.202 \ldots$ is Apery's constant and $N$ is the number of atoms in the crystal, as in Fig. 1. They find

$$
\operatorname{Var}\left[b_{\ell}(\ell \tau+x)-b_{\ell}(\ell \tau)\right] \cong(\mathcal{A} \ell)^{2 / 3} g\left(\mathcal{A}^{1 / 3} \ell^{-2 / 3} x\right),
$$

where $\mathcal{A}=(1 / 2) b_{\infty}^{\prime \prime} 27$. This leads to the central result that the width $w \sim \ell^{1 / 3}$. Furthermore, the fluctuations are non-Gaussian. They also show that near the shoreline, the deviation of the equilibrium crystal shape from the facet plane takes on the Gruber-Mullins-PokrovskyTalapov [28 form $-\left(r-r_{0}\right)^{3 / 2}$, where $r$ is the lateral distance from the facet center and $r_{0}$ is the radius of the facet.

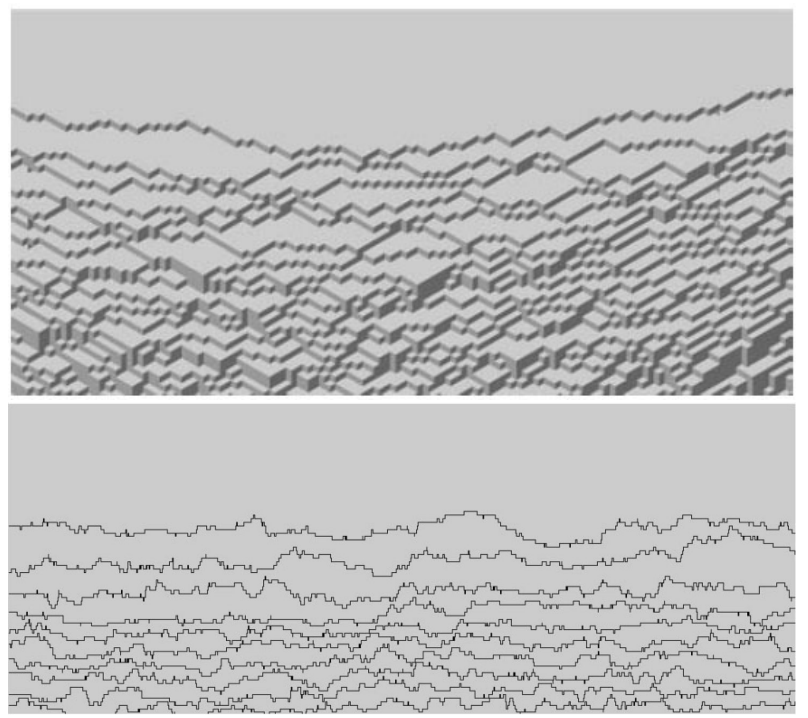

FIG. 3. a) Magnified detail of the steps near the facet edge in Fig. 1. from Refs. 15, 16]; b) Snapshot of computed configurations of the top steps (those near a facet at the flattened side portion of a cylinder) for a terrace-step-kink (TSK) model with volume constraint, from Refs. [14, 16].

\section{ANALYTICAL RESULTS}

In this section, we discuss computation of the time scaling of step-edge fluctuations using two non-rigorous approaches. First, we adopt a simple scaling argument, starting from FPS's exact result. Then we derive a continuum-equation description of the step bordering a crystal facet. Then, with simple power counting we rederive FPS's result, as well as the temporal power-law scaling of edge fluctuations.

For straight steps, which underlie treatments of this problem, one adopts cartesian coordinates $(x, y), y$ being in the direction along the step edge, and $x(y)$ describing the step profile, in what has been called "Maryland notation" 29]. We focus attention on the step autocorrelation function

$$
G(t)=\left\langle\left[x\left(y_{0}, t+t_{0}\right)-x\left(y_{0}, t_{0}\right)\right]^{2}\right\rangle_{y_{0}, t_{0}} \underset{t \rightarrow 0}{\sim} t^{2 \beta},
$$

which can readily be computed in a Monte Carlo simulation [8] and measured experimentally with a scanning probe like STM. It is less feasible to measure spatial correlation functions since such experiments do not take an instantaneous "snapshot." Like a television screen, different parts of the micrograph correspond to different times, and it is problematic to deal with what transpires between successive visits by the STM tip to nearby positions. Furthermore, in such experiments one does not do a full average over $y_{0}$ but rather picks a single value; for that case we replace $G(t)$ by $G\left(y_{0}, t\right)$, for which there is no spatial average. The resulting plot of displacement $x$ vs. time looks similar to scans of $x$ along a step, and so are called "pseudo-images" 2. (Cf. Fig. 6 below.) At short times $G\left(y_{0}, t\right)$ exhibits the same $t^{2 \beta}$ behavior as $G(t)$. The value of exponent $\beta$ depends on the atomistic processes responsible for the fluctuations of the step, but also on the position of the step with respect to a crystal facet, as we proved in Ref. 20 and recount shortly below. In the other limit, $G(t \rightarrow \infty)$ saturates to $2 w^{2}$, where $w$ is width of the fluctuations.

When doing simulations 23] (or if one had a probe that could take instantaneous snapshots), one can probe

$$
G\left(y, t_{0}\right)=\left\langle\left[x\left(y_{0}+y, t_{0}\right)-x\left(y_{0}, t_{0}\right)\right]^{2}\right\rangle_{y_{0}} \sim w^{2},
$$

for large values of $y$. Then the roughness exponent $\alpha$ can be extracted from the saturation value of the width $w$ of the fluctuating step by using Eq. (1) and identifying $E$ with the size of the system in the $y$-direction.

\section{A. Heuristic Derivation}

Starting from FPS's result that the roughness exponent $\alpha=1 / 3$ in Eq. (1), we apply Pimpinelli et al.'s argument [18] for finding the temporal scaling: Referring to Fig. 4. consider a protruding portion of step of length $E$ and width $w$. At equilibrium, the protrusion is 


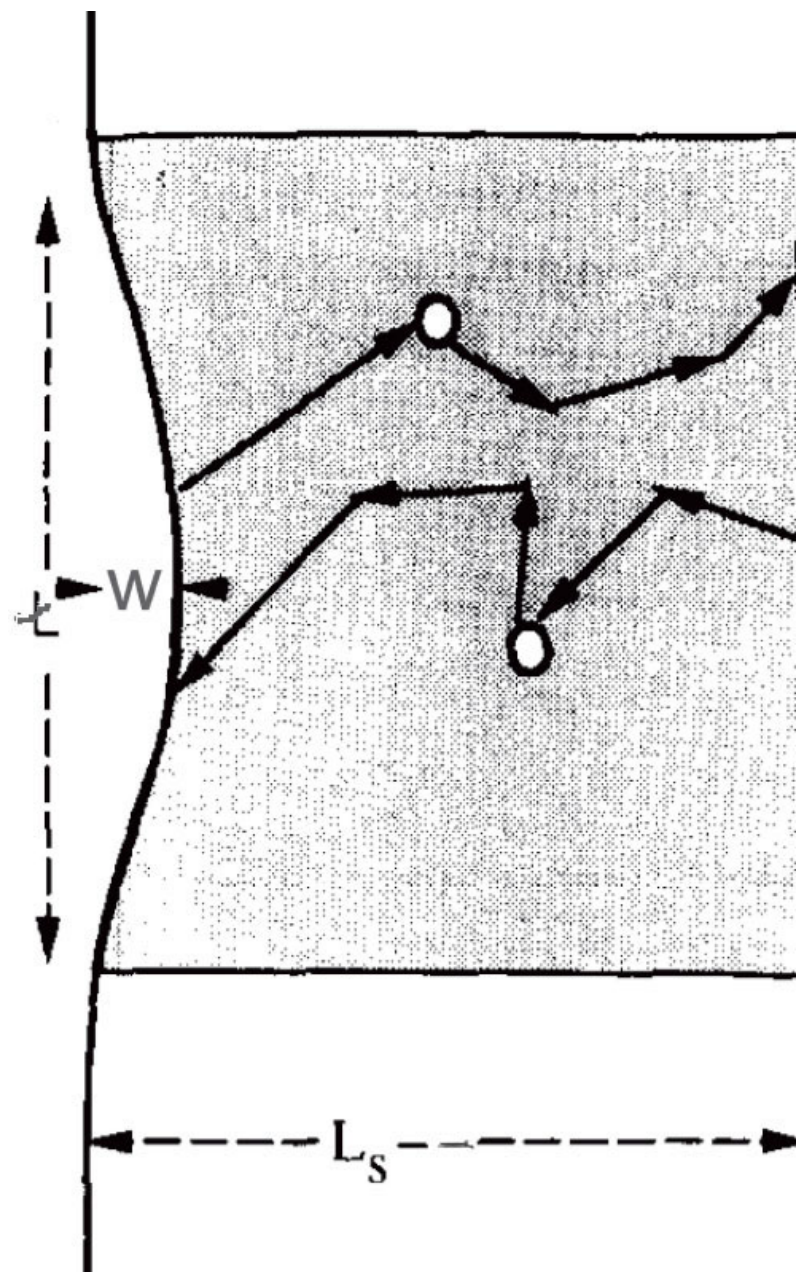

FIG. 4. Schematic of a stepped surface seen from above, to illustrate the "pipe" concept of Ref. [18]. The fluctuating step, depicted as a thick wavy line, shows the definitions of $w$ and $E$. For the two particular cases considered in the text, the thickness $L_{s}$ of the pipe-like reservoir to the right of the step, in which atom exchange occurs, is of order the lattice spacing a. Adapted from Ref. [18].

due to fluctuations in the number of atoms to and from a "reservoir. The reservoir is assumed to be, depending on the limiting atom transport process, either another part of the same step, or a region on the crystal surface. Before considering a specific kinetic process, let us derive a few general relations. On average, a number $N(t)$ of atoms continually move between the step and the reservoir during a time interval $t$. The reservoir is by definition situated one diffusion length $L_{s}$ away from the protrusion. While the net flux to and from the step vanishes, the number $N(t)$ fluctuates around its vanishing mean; assuming that atom fluxes in different time intervals are uncorrelated, we can compute the typical size of the fluctuation $\delta N$, which is of order $\sqrt{N(t)}$. Denoting by $\Omega$ the atomic area, we estimate the size $w$ of the protrusion along the step edge (defined, as said above, as the amplitude or width of a typical step fluctuation of length
E) from $w \times E \approx \Omega \sqrt{N(t)}$. To estimate $N(t)$, we note that the size (surface area) of the reservoir feeding the fluctuation is $E L_{s}$. Then the number of atoms moving to and from the step edge during time $t$ is proportional to the number of diffusing atoms in the region feeding the step, $c_{e q} E L_{s}$ (where $c_{e q}$ is the equilibrium particle density) and to the fraction of time the atoms spend in this region, $t / \tau^{*}$. The characteristic time $\tau^{*}$ depends on the specific transport process (see below). Then, as in Ref. [18]:

$$
N(t) \approx \frac{c_{e q}}{\tau^{*}} E L_{s} t
$$

Furthermore, the squared area of the fluctuating bulge is

$$
w^{2} E^{2} \approx(\delta N)^{2} \approx N
$$

where we assume $\delta N \approx \sqrt{N}$.

While Ref. 18 considers several different cases, we focus here on the two primary scenarios: i) nonconservative mass transport by attachment-detachment to/from the step edge, with fast terrace diffusion (A/D) and ii) conservative mass transport by step-edge diffusion (SED).

i) In the $\mathrm{A} / \mathrm{D}$ case, $1 / \tau^{*} \approx k$, where $k$ is an appropriate kinetic coefficient. For fast surface diffusion, the step effectively exchanges atoms with a "2D adatom vapor" on the surface. Then, $L_{s}$ is of order the lattice spacing $a$, and Eq. (9) yields

$$
w^{2} \iota^{2} \approx k c_{e q} \text { Eat. }
$$

Using Eq. (2) leads to $E^{5 / 3} \sim t$, and eventually to

$$
w \sim t^{1 / 5} \rightarrow G(t) \sim t^{2 / 5}
$$

In comparison, $G(t) \sim t^{1 / 2}$ for a straight step or an isolated 2D island [9, 18.

ii) For mass transport by step-edge diffusion along a portion of step of size $E, 1 / \tau^{*} \approx D_{e} / E^{2}$, where $D_{e}$ is the edge diffusion coefficient. Again in this case $L_{s} \approx a$, so that Eq. 9 becomes

$$
N(t) \approx t c_{e q} D_{e} a / t
$$

From Eq. 10

$$
w^{2} \approx t c_{e q} D_{e} a / E^{3}
$$

With Eq. 22 we now find $t \sim t^{11 / 3}$, so that

$$
w \approx t^{1 / 11} \rightarrow G(t) \sim t^{2 / 11} .
$$

$(\beta=1 / 11)$ for a crystal facet fluctuating through step edge diffusion. In comparison, $G(t) \sim t^{1 / 4}(\beta=1 / 2)$ for a straight step or an isolated (large) 2D island [9, 18. 


\section{B. Scaling in Curved Geometry with Asymmetric Potential}

We have also considered this problem from the perspective of scaling theory. This approach begins by writing a Langevin equation for the motion of the edge of a crystal facet, or of an island. In polar coordinates, $r(\theta, t)$, the facet/island radius (the position of the edge) in direction $\theta$ and at time $t$, satisfies the stochastic differential equation

$$
\frac{\partial r(\theta, t)}{\partial t}=f\left[r(\theta, t), r_{\theta}, r_{\theta \theta}, \ldots\right]+\eta(\theta, t),
$$

where $r_{\theta} \equiv \partial r / \partial \theta, r_{\theta \theta} \equiv \partial^{2} r / \partial \theta^{2}$, and so on for higherorder derivatives. The function $f$ describes the deterministic relaxation of fluctuations and depends in principle on the facet radius and its higher-order derivatives with respect to $\theta$. Finally, $\eta(\theta, t)$ is a white noise, which is conservative or non-conservative for SED or $\mathrm{A} / \mathrm{D}$, respectively.

To obtain $f$ we assume that the facet/island is delimited by a closed step of free energy per length $\beta(\theta)$. Neglecting step-step interactions (as in Ref. [14]), the free energy of the facet/island is

$$
F=\int_{0}^{2 \pi} \beta(\vartheta) \sqrt{r^{2}+r_{\theta}^{2}} d \theta
$$

where $\vartheta$ is the local direction of the step 30.

Assuming for simplicity (as in Ref. [14]) an isotropic step free energy $\beta(\theta)=\beta$ (implying a circular facet or island) one can compute straightforwardly the excess chemical potential with respect to a perfectly circular step edge, which is given by the Gibbs-Thomson relation (see 30,32 )

$$
\delta \mu=\Omega \beta\left(\frac{r^{2}-r r_{\theta \theta}+2 r_{\theta}^{2}}{\left(r^{2}+r_{\theta}^{2}\right)^{3 / 2}}-\frac{1}{\rho_{0}}\right)
$$

where the first term in the parentheses is the step curvature and $\rho_{0}$ the average facet/island radius.

In order to study fluctuations around $\rho_{0}$, it is useful to introduce the new variable $\tilde{r}(\theta, t)=\left[r(\theta, t)-\rho_{0}\right] / \rho_{0}$. In terms of this variable, the excess chemical potential reads

$$
\delta \mu=\frac{\Omega \beta}{\rho_{0}} \frac{(1+\tilde{r})\left(1+\tilde{r}-\tilde{r}_{\theta \theta}\right)+2 \tilde{r}_{\theta}^{2}}{\left[(1+\tilde{r})^{2}+\tilde{r}_{\theta}^{2}\right]^{3 / 2}}-\frac{\Omega \beta}{\rho_{0}} .
$$

Expanding around $\tilde{r}=0$, discarding all terms in $\tilde{r}$ as much smaller than unity but keeping the lowest nonlinear terms in the derivative $\tilde{r}_{\theta}$, we obtain

$$
\delta \mu \approx \frac{\Omega \beta}{\rho_{0}}\left(-\tilde{r}_{\theta \theta}+\frac{1}{2} \tilde{r}_{\theta}^{2}\right) .
$$

With the chemical potential in hand, we can model the step-edge fluctuations as a Langevin equation for the two limiting cases considered previously. (In experiments on real crystals, of course, one can observe either of these two limiting behaviors or both, as well as crossover between them, depending on observational time scales and temperature. In metals, for instance, SED is known to dominate over $\mathrm{A} / \mathrm{D}$ at low temperature, when thermal energy is not large enough to allow atoms to detach from a step edge. Therefore, A/D will only become observable at long times, with temperature determining how long a "long time" is.) i) In A/D atoms "evaporate from" and "condense into" the step edge. Accordingly, we write 20, 31, 32]

$$
\frac{\partial \tilde{r}(\theta, t)}{\partial t}=-\frac{\Gamma_{A D}}{k_{B} T} \delta \mu\left(\tilde{r}, \tilde{r}_{\theta}, \tilde{r}_{\theta \theta}\right)+\eta(\theta, t)
$$

where $\Gamma_{A D}$ is the attachment-detachment kinetic coefficient, and $\eta(\theta, t)$ is a Gaussian white noise. Inserting Eq. 20 into Eq. 21) we find

$$
\frac{\partial \tilde{r}(\theta, t)}{\partial t}=\frac{\Gamma_{A D}}{k_{B} T} \frac{\Omega \beta}{\rho_{0}^{3}}\left[\frac{\partial^{2} \tilde{r}}{\partial \theta^{2}}-\frac{1}{2}\left(\frac{\partial \tilde{r}}{\partial \theta}\right)^{2}\right]+\eta(\theta, t) .
$$

ii) Similarly for SED, we use conserved dynamics to represent atomic diffusion along the step edge. Accordingly, we write 20, 31, 32,

$$
\frac{\partial \tilde{r}(\theta, t)}{\partial t}=\frac{\Gamma_{S E D}}{k_{B} T \rho_{0}^{2}} \frac{\partial^{2}}{\partial \theta^{2}} \delta \mu\left(\tilde{r}, \tilde{r}_{\theta}, \tilde{r}_{\theta \theta}\right)+\eta_{C}(\theta, t)
$$

where $\Gamma_{S E D}$ is the step-edge -diffusion kinetic coefficient, and $\eta_{C}(\theta, t)$ is a conserved Gaussian white noise [33]. Inserting Eq. 20 into Eq. (23), we get

$$
\frac{\partial \tilde{r}(\theta, t)}{\partial t}=\frac{\Gamma_{S E D}}{k_{B} T} \frac{\Omega \beta}{\rho_{0}{ }^{3}}\left[-\frac{\partial^{4} \tilde{r}}{\partial \theta^{4}}+\frac{1}{2} \frac{\partial^{2}}{\partial \theta^{2}}\left(\frac{\partial \tilde{r}}{\partial \theta}\right)^{2}\right]+\eta_{C}(\theta, t) .
$$

Eqs. 22 and 24) resemble the Kardar-Parisi-Zhang (KPZ) 34 equation and its conserved counterpart (the "Montreal model") 35, respectively. Here, however, the non-linear terms come from the equilibrium curvature of the interface, while they are induced by nonequilibrium effects in the KPZ and Montreal models. A closed (curved) step, such as the border of a facet or an island, has an "inside" and an "outside". The chemical potential in Eq. 19 discriminates between inward and outward fluctuations of the step radius around its mean value $\rho_{0}$, thus breaking the radial symmetry. This is the origin of the "asymmetric" KPZ-like nonlinearity in Eqs. 22 and (24), which indeed breaks the $\tilde{r} \rightarrow-\tilde{r}$ symmetry of the equations. The asymmetry is insufficient to change the universality class of the fluctuating step. We will see that KPZ or Montreal exponents (see, e.g., Ref. [36]) are still expected for the fluctuations of the edge of a small-enough (viz., smaller than the capillary length $k_{B} T / \beta$ ) island. In contrast, a facet should exhibit the exponents that we have computed in the previous subsection, which are neither KPZ nor Montreal. 
The difference stems from the interactions of the fact edge with the neighboring steps, which limit the amplitude of the fluctuations and change the character of the noise term in Eqs. (22) and (24). In the previous subsection, the effect of interactions was implicitly introduced through the assumption that the exponent $\alpha=1 / 3$. In the following, we derive the latter, too, using a scaling argument. Step-step interactions constitute another source of asymmetry at a facet edge, since steps are only present on one side of the fluctuating step. We will investigate the interaction-induced asymmetry in Section 5 .

To illustrate how our scaling arguments work, we first consider fluctuations of a straight step in the A/D case. (The rescaling in angle assumes that the angle is small, so that it essentially parametrizes distance along the arc.) The step-edge fluctuations (in the A/D case) obey the linear equation 31, 32]

$$
\frac{\partial x(y, t)}{\partial t}=\frac{\Gamma_{A D} \Omega \beta}{k_{B} T} \frac{\partial^{2} x}{\partial y^{2}}+\eta(y, t) .
$$

Rather than simply solving this linear equation, we use it to illustrate the scaling argument. Assume that the linear size $E$ along the step edge is dilated by a factor $b, E^{\prime}=b \pm$ (where primed variables denote rescaled quantities). Scaling implies that the width $w$ of a fluctuation varies as $w \sim E^{\alpha}$, so that $w^{\prime}=b^{\alpha} w$. (See, e.g., Ref. [36].) The typical time needed to develop a fluctuation of size $E$ scales as $t \sim E^{z}$, so that $t^{\prime}=b^{z} t$. The time derivative in Eq. 25) scales then as

$$
\frac{\partial x^{\prime}\left(y^{\prime}, t^{\prime}\right)}{\partial t^{\prime}}=b^{\alpha-z} \frac{\partial x(y, t)}{\partial t},
$$

while the Laplacian term scales as

$$
\frac{\partial^{2} x^{\prime}\left(y^{\prime}, t^{\prime}\right)}{\partial y^{\prime 2}}=b^{\alpha-2} \frac{\partial^{2} x(y, t)}{\partial y^{2}} .
$$

Then equating the scaling exponents of $b$ in Eqs. (26) and (27) yields $z=2$.

The scaling exponent $\alpha$ depends on the scaling behavior of the noise term in the problem of interest. If the step is isolated, the step edge should be treated as a $1 \mathrm{D}$ interface. Then, since $\eta\left(y_{1}, t_{1}\right) \eta\left(y_{2}, t_{2}\right)=\delta\left(y_{1}-y_{2}\right) \delta\left(t_{1}-t_{2}\right)$, the noise term scales as

$$
\eta^{\prime}\left(y^{\prime}, t^{\prime}\right)=b^{-(1+z) / 2} \eta(y, t) .
$$

Equating the scaling exponents of $b$ in Eqs. (26) and (28) and using $z=2$ gives the random-walk value

$$
\alpha=1 / 2 .
$$

These results are tabulated in the first line of Table \. The second line gives analogous results for the SED case.

If the step is inside a train, as on a vicinal surface, then Eq. 25) must be replaced by a similar second-order equation for the surface profile $z=z(x, y)$. With $x$ and $y$ chosen so that they are perpendicular and parallel to the average step direction, respectively, the Laplacian in Eq. (25) is replaced by second derivatives of $z$ with respect to $x$ and $y$ with different coefficients, reflecting that the stiffness of a vicinal surface differs in directions parallel and perpendicular to the steps. The scaling of these terms, however, does not change with respect to Eq. 27). The important point is that in this case the fluctuations take on a $2 \mathrm{D}$ character, so that the noise term scales as

$$
\eta^{\prime}\left(x^{\prime}, y^{\prime}, t^{\prime}\right)=b^{-(2+z) / 2} \eta(x, y, t) .
$$

Equating the scaling exponents of $b$ in Eqs. 26) and 28) and using $z=2$ now yields $\alpha=0$, corresponding to the expected logarithmic scaling 23] $w \sim \ln E$.

We turn now to the topic of primary concern, the scaling behavior of nonlinear Eqs. (22) and (24) for a facet edge, for which the nonlinearity dominates the scaling. This nonlinearity comes from the curvature of the step edge (Gibbs-Thomson effect), but the fluctuation spectrum may differ differ in a subtle way from the analogous term in the KPZ equation. The latter is an equation for a growing interface, which roughens during growth. Because of the nonlinear term, the noise-induced roughening of the interface cannot be captured by a simple power-counting argument such as we used for the linear Eq. (25). Indeed, except for the 1D case, the scaling of the KPZ equations is still an open problem. Here, we are addressing equilibrium fluctuations of the interface; we can expect that their scaling will differ from the growth case. In fact, the different physical situations represented by an island edge and by a facet shoreline suggest that the noise term has to be treated differently in the two cases. Ultimately, the difference stems from the fact that, unlike the boundary step of a facet, an island edge is free to fluctuate, the amplitude $w$ of its fluctuations being limited only by the size of the island. Because of the hindrance of neighboring steps, the fluctuations of a facet are constrained to smaller amplitudes than those of an island of comparable size. As noted above, the non-linear term becomes important only for small (relative to the capillary length) islands. However, the radius of an island has to be larger than a minimum value in order for the island to be stable [37. More details can be found elsewhere [24, 38, 39].

\begin{tabular}{|l||c|c|c|c||c|c|}
\hline Class & $\partial / \partial t$ & $\mathrm{~L} \nabla^{2,4}$ & NL KPZ & Noise & $\alpha$ & $z$ \\
\hline Isolated A/D & $\alpha-z$ & $\alpha-2$ & - & $-(1+z) / 2$ & $1 / 2$ & 2 \\
\hline Isolated SED & $\alpha-z$ & $\alpha-4$ & - & $-(3+z) / 2$ & $1 / 2$ & 4 \\
\hline Train A/D & $\alpha-z$ & $\alpha-2$ & - & $-(2+z) / 2$ & $0(\ln )$ & 2 \\
\hline \hline Asym A/D & $\alpha-z$ & $\alpha-2$ & $2 \alpha-2$ & $-(1+z) / 2$ & $1 / 3$ & $5 / 3$ \\
\hline Asym SED & $\alpha-z$ & $\alpha-4$ & $2 \alpha-4$ & $-(3+z) / 2$ & $1 / 3$ & $11 / 3$ \\
\hline
\end{tabular}

TABLE I. Summary of exponents resulting from scaling arguments for the evolution, [linear] relaxation, and noise terms of the relevant Langevin equations, as well as the nonlinear KPZ term for facet edges. The deduced values of the roughness exponent $\alpha$ and the dynamic exponent $z$ are then listed. See text for details. 
The main conclusion is that the step edge bordering an island may have larger-amplitude fluctuations than does the facet edge, since the latter is limited by the presence of the neighboring steps. (Likewise, since these steps are only on one side, the facet edge has larger fluctuations than a step in the middle of a step train.) Thus, the noise terms scale differently for a facet and for an island, leading to different temporal and spatial scaling behaviors. Again $w$ is the width of a step-edge protrusion of size $E$. In order to proceed as simply as possible, we decided to resort to the approach in Hentschel and Family [40, which was shown to yield the exact scaling in 1D and a very good approximation in higher dimensions. In this approach, one takes the length $S$ of a step fluctuation of amplitude $w$ to vary as $S \approx\left(w^{2}+E^{2}\right)^{1 / 2} \sim E$ or $w$ for amplitude fluctuations that are small or large, respectively. Assuming that atoms are added (or removed) randomly to the step edge (for either A/D or SED), the relative fluctuations of the length of the edge are just $\Delta S / S \approx S^{-1 / 2}$, indicating that it is reasonable to assume that the noise term in our stochastic equations scales as

$$
\eta(S, t) \sim(S t)^{-1 / 2} .
$$

As a consequence, for fluctuations of small amplitude we have

$$
\eta\left(S^{\prime}, t^{\prime}\right) \sim\left(E^{\prime} t^{\prime}\right)^{-1 / 2} \sim b^{-(1+z) / 2} \eta(S, t),
$$

while for large amplitude

$$
\eta\left(S^{\prime}, t^{\prime}\right) \sim\left(w^{\prime} t^{\prime}\right)^{-1 / 2} \sim b^{-(\alpha+z) / 2} \eta(S, t) .
$$

The last comment concerns the scaling variable. In Eqs. (22) and (24) the variable is an angle, but since the facet (island) radius $\rho_{0}$ is kept fixed, the quantity $\rho_{0} \theta$ measures the length along the step edge, and scales as a length. We can now address the scaling. Consider first a facet fluctuating by attachment-detachment, Eq. (21). In this case, step fluctuations are limited in amplitude by neighboring steps. Equating the scaling of the time derivative to that of the noise term in Eq. (32) yields

$$
z=2 \alpha+1 \text {. }
$$

The nonlinear term $\tilde{r}_{\theta}^{2}$ scales as

$$
\left(\frac{\partial \tilde{r}^{\prime}}{\partial \theta^{\prime}}\right)^{2}=b^{2 \alpha-2}\left(\frac{\partial \tilde{r}}{\partial \theta}\right)^{2}
$$

Equating Eq. (35) to the noise term Eq. 32 yields

$$
4 \alpha+z=3
$$

From Eqs. 34 and (36) we finally get FPS's result

$$
\alpha=1 / 3 \quad \Rightarrow \quad \tilde{r} \sim E^{1 / 3} .
$$

The dynamic scaling of step fluctuations turns out to be what we computed previously: From Eqs. (34) and (37), e.g., we obtain

$$
z=5 / 3 \quad \Rightarrow \quad \alpha / z=\beta=1 / 5,
$$

which, recalling that $G(t) \sim t^{2 \beta}$, coincides with Eq. 12.

Facet fluctuations driven by step-edge diffusion obey Eq. (24). The conserved noise term scales as

$$
\eta_{C}^{\prime}\left(S^{\prime}, t^{\prime}\right)=b^{-(3+z) / 2} \eta_{C}(S, t)
$$

The conserved nonlinear term scales now as

$$
\frac{\partial^{2}}{\partial \theta^{\prime 2}}\left(\frac{\partial \tilde{r}^{\prime}}{\partial \theta^{\prime}}\right) 2=b^{2 \alpha-4} \frac{\partial^{2}}{\partial \theta^{2}}\left(\frac{\partial \tilde{r}}{\partial \theta}\right)^{2}
$$

Equating (39) and 40 yields

$$
4 \alpha+z=5
$$

Equating the time derivative to the noise term Eq. 39 yields

$$
2 \alpha=z-3
$$

Together Eqs. 41) and 42 yield

$$
\alpha=1 / 3, \quad z=11 / 3 \quad \Rightarrow \quad \beta=1 / 11
$$

as in Eq. (15).

As mentioned above, KPZ-like or Montreal-like exponents are expected to appear in the fluctuations of a small island edge, for non-conserved and conserved dynamics, respectively. For the latter, fluctuations are not hindered, and the noise scales as in Eq. (32). It then follows straightforwardly that the scaling relations $\alpha+z=2$ and $3 \alpha=z$, implying $\alpha=1 / 2$ as for a random walk [cf. Eq. [29] ], as well as the KPZ result (first noted in Ref. 20]) that

$$
\beta=1 / 3
$$

This result clearly applies only to small islands with large curvature; otherwise, the scaling should be that of a straight step.

Table II] summarizes the various universality classes 36, 40, that can arise for different types of spatial confinement for non-conserved and conserved kinetics .

Finite-volume effects on supported nano-crystallites with a Gruber-Mullins-Pokrovsky-Talapov surface free energy density [28] have been found to produce metastable states with different crystal shapes [42 for a given crystal-substrate interface boundary condition [12, 43. All shapes have a facet smoothly connected to a vicinal region, which obeys an $x^{3 / 2}$ shape power law in equilibrium 44. Once a crystallite attains a stable state, the step that serves as the interface between the facet and the vicinal region (see Fig. 1b)) fluctuates around its stable position, which is determined by the asymmetric potential established by step-step interactions and the "reservoir" chemical potential of the crystallite. 


\begin{tabular}{l||l|c|c|c||c|c|c|c} 
Geom. & $\mathrm{A} / \mathrm{D}$ & $\alpha$ & $2 \beta$ & $z$ & $\mathrm{SED}$ & $\alpha$ & $2 \beta$ & $z$ \\
\hline Free & $l \mathrm{M}_{1}^{2}(\mathrm{EW})$ & $\frac{1}{2}$ & $\frac{1}{2}$ & 2 & $l \mathrm{C}_{1}^{4}$ & $\frac{1}{2}$ & $\frac{1}{4}$ & 4 \\
Sym-cfn & $l \mathrm{M}_{2}^{2}$ & 0 & 0 & 2 & $l \mathrm{C}_{2}^{4}$ & 0 & 0 & 4 \\
Asy-cfn & $\mathrm{KYP}[41]$ & $\frac{1}{3}$ & $\frac{2}{5}$ & $\frac{5}{3}$ & $n \mathrm{C}_{1}^{4}$ & $\frac{1}{3}$ & $\frac{2}{11}$ & $\frac{11}{3}$ \\
\hline $\mathrm{KPZ}$ & $n \mathrm{~N}_{1}^{2}$ & $\frac{1}{2}$ & $\frac{2}{3}$ & $\frac{3}{2}$ & $n \mathrm{M}_{2}^{4}$ & $\frac{2}{3}$ & $\frac{2}{5}$ & $\frac{10}{3}$
\end{tabular}

TABLE II. Summary of the dynamical scaling universality classes for crystallite steps. The geometries included are: Free $=$ an isolated step or island edge, Sym-cfn = steps symmetrically confined by the nearby steps as in a step bunch, and Asy-cfn = steps confined by an asymmetric potential, esp. a facet edge. The KPZ class is included for comparison. In the underlying Langevin equation (cf. Ref. [36]), $l$ or $n$ indicates whether the equation is linear or non-linear (has a KPZ term). $\mathrm{C}$ or $\mathrm{N}$ indicates whether the deterministic part and the noise are conservative or non-conservative; $\mathrm{M}$ denotes mixed, with the former conservative but the noise not. The superscript (2 or 4) indicates the power of $\nabla$ in the linear conservative term, while the subscript gives the dimensionality of the independent variable. From Ref. 19 .

\section{a) isolated}

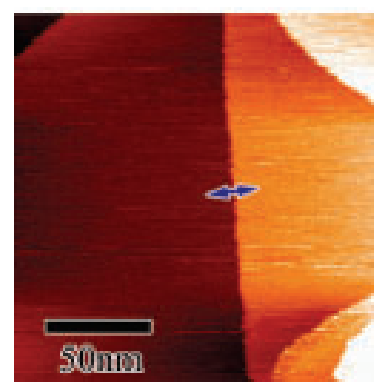

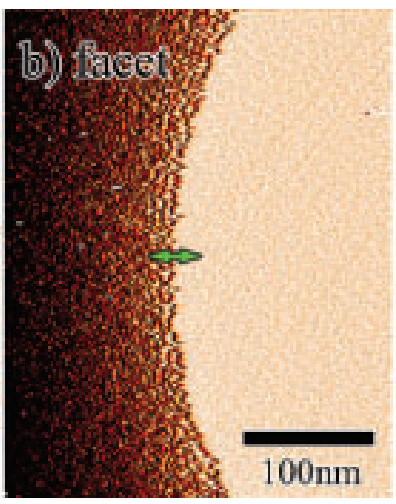

FIG. 5. An STM image of a) an isolated step on a crystallite facet (room temperature) and b) a crystal facet edge (350K). The small superimposed double-arrow indicates the tip path that leads to line-scan images as in Fig. 6. From Refs. [19, 39].

\section{STM EXPERIMENTS}

The first experimental observations of the novel scaling predicted for facet-edge fluctuations on crystallites were performed at the University of Maryland by Masashi Degawa working under Ellen D. Williams [19]. Crystallites were formed by depositing a $20-30 \mathrm{~nm} \mathrm{~Pb}$ film at room temperature on a $\mathrm{Ru}(0001)$ substrate in UHV [45], and subsequently dewetting at $620 \mathrm{~K}$. The liquid $\mathrm{Pb}$ droplets solidified upon slow cooling and were left to equilibrate to a stable state at the $T$ of the experiment [25, 45, 46]. The crystallites were observed with a variable-temperature scanning tunneling microscope (VT-STM) after equilibration. Figure 5 depicts an STM image of a) an isolated step (at room temperature) and b) facet-edge (at 350K). A crystallite in a stable state as shown in b) has a flat, close-to-circular (111) facet and a smoothly-connecting vicinal region.

Since STM images are scans rather than instantaneous
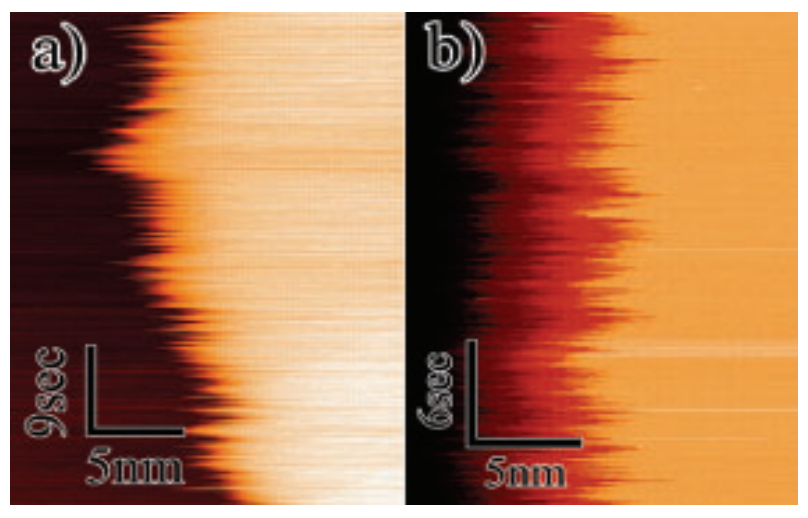

FIG. 6. Segment of a line-scan pseudoimage of a) an isolated step (step from screw dislocation) and b) a facet-edge at $350 \mathrm{~K}$, showing also the correlated fluctuations of the neighboring steps. The time interval between lines is $0.02 \mathrm{~s}$, and 2000 lines are measured per image. From Refs. [19, 39.

"snapshots", the data for dynamic scaling can be more compelling than that for static scaling. By repeatedly scanning perpendicularly to a single position $\left(y_{0}\right)$ along the facet-edge or step (cf. Fig. 5), we obtain a line-scan STM image [2] $x(t)$, as shown in Fig. 6for a) an isolated step (step from a screw dislocation) and b) a facet-edge, both at 350K. Digitized step displacement-positions $x(t)$ extracted from these "pseudoimages" are used for statistical analysis. To evaluate the growth exponent $\beta$, we calculated the early behavior of the time correlation function $G(t)$ given in Eq. (7). To evaluate the roughness exponent $\alpha$, we can calculate either the saturation value of the width $w$ of the fluctuating step as in Eq. (1) or the spatial correlation function $G\left(y, t_{0}\right) \sim y^{2 \alpha}$ for $y$ less than the correlation length [23].

Figure 7 shows $G(t)$ determined for a) facet-edges and b) isolated step-edges. Squares, circles, and triangles represent measurements at $300 \mathrm{~K}, 350 \mathrm{~K}$ and $400 \mathrm{~K}$, respectively. Each curve displays the average over the correlation functions for 10-30 measurements of $x\left(y_{0}, t\right)$. The exponent $2 \beta$ for each temperature is obtained from the slope of the curve on the log-log plot; the values of these slopes are listed in the figure caption. As expected the exponents show no systematic thermal dependence: from all data sets, the $\sigma^{-2}$-weighted average exponent is $2 \beta=0.149 \pm 0.032$ for facet-edges and $2 \beta=0.262 \pm 0.021$ for isolated steps. With over $99.9 \%$ confidence (using Student's t-test), these values come from different parent populations. Each of the two results is within one standard deviation, $\sigma$, of their respective predicted values of $2 / 11$ and $1 / 4$.

Determination of the roughness exponent $\alpha$ requires evaluation of the system-size dependence. A detailed examination is a challenge beyond the capability of the STM experiments being used. However, we can demonstrate that size does affect fluctuations. Under the assumption $E \sim R$ (the facet radius) for confined steps (Fig. 7a), we expect $w^{2} \sim R^{2 \alpha}$. (For the unconfined 

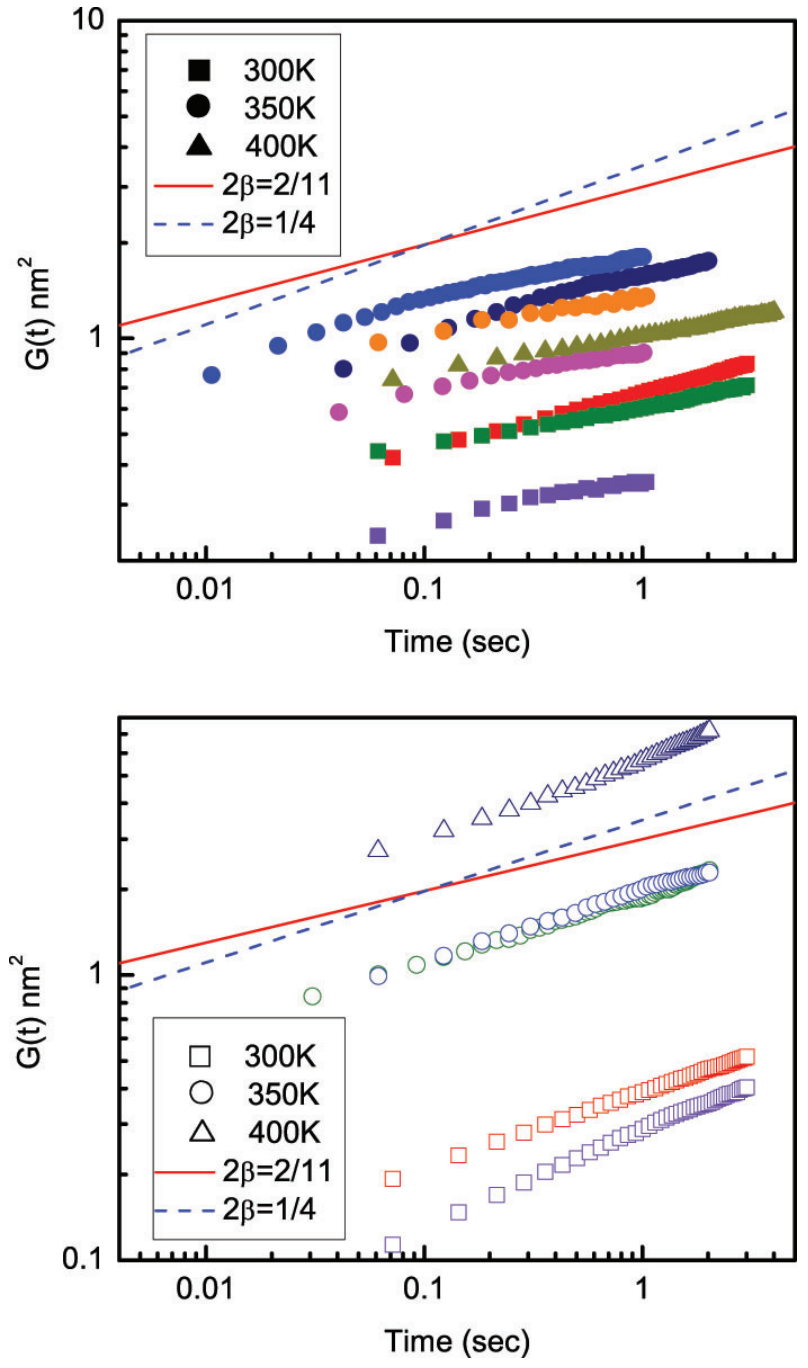

FIG. 7. Log-log plot of $G\left(\left[y_{0},\right] t\right)$ (cf. Eq. (7)) for a) facet edges and b) isolated steps with facet radii from 60 to 190 $\mathrm{nm}$. The symbols represent: $300 \mathrm{~K}$ (squares), 350K (circles), and 400K (triangles). For guidance, solid and dashed lines show slopes $2 / 11=0 . \overline{18}$ and $1 / 4$, respectively. Individual fits to each of the data sets yield slopes of a) facet edges: 300K: 0.18(1), 0.13(6), 0.13(2); 350K: 0.17(4), 0.17(4), $0.12(3), 0.11(5) ; 400 \mathrm{~K}: 0.12(12)$, and b) isolated steps: $300 \mathrm{~K}$ : 0.32(3), 0.26(1); 350K: 0.24(3), 0.24(4); 400K: 0.30(4). From Refs. 19, 39.

steps, the system size is larger than the limitations imposed by the finite measurement time [4].) The effect of the facet size is apparent in Fig. $7 \mathrm{a}$ since the three upper sets of data at $350 \mathrm{~K}$ were taken on larger crystallites, having radii greater than $100 \mathrm{~nm}$. More quantitatively, Fig. 8 plots the characteristic length $w^{2} \tilde{\beta} / k_{B} T$ vs. facet radius at $300 \mathrm{~K}$ and $350 \mathrm{~K}$, using for the step stiffness $\tilde{\beta}$ the values $0.339 \mathrm{eV} / \mathrm{nm}$ and $0.327 \mathrm{eV} / \mathrm{nm}$ [47, respectively. Fits to the data yield exponents within the predicted range of $\alpha=1 / 3$ (solid) to $\alpha=1 / 2$ (dash). Although there are insufficient data to distinguish between these two values [48, the results do show that $R$ influences the fluctua-

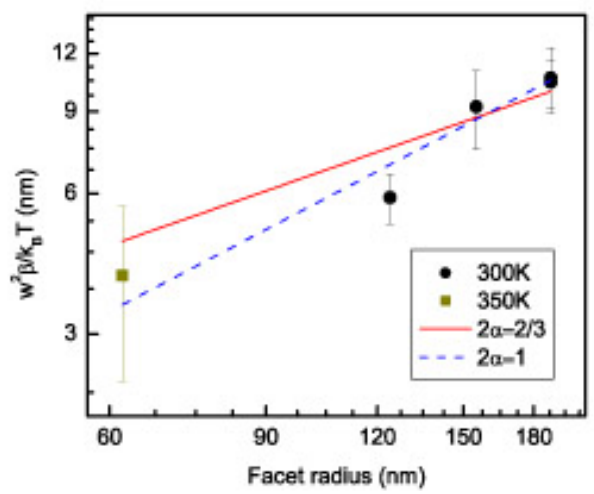

FIG. 8. Product of squared saturation width and reduced stiffness as a function of facet radius (facet-edge only). Circles and squares are room temperature and $350 \mathrm{~K}$, respectively. Solid and dashed lines are a fit to the $350 \mathrm{~K}$ data with $\alpha=1 / 3$ and $\alpha=1 / 2$, respectively. From Refs. [19, 39].

tions, providing further evidence that effects of crystal confinement govern the behavior of $G(t)$.

The fluctuations of facet edges evidently belong to a different universality class of dynamic scaling from that of an isolated step on a surface. In contrast to previous predictions for step exponents 1, 2, 49, 50, this difference does not stem from the type of kinetics. Instead, the effect is predicted to result from the coupling of the step chemical potential to the fluctuations: For facetedge fluctuations the step confinement is produced by an increase in local step chemical potential $\mu(x)$ when the step is displaced from equilibrium. The functional behavior of $\mu(x)$ results from a competition between the step-repulsions from the vicinal region and the $2 \mathrm{D}$ pressure of the adatom density on the facet, which in turn stems from the constraints governing the crystallite shape [12, 42]. For a step symmetrically confined on a vicinal surface, the confinement corresponds to a potential that is quadratic in displacement [51. However, for the facet-edge step, the asymmetry in the $\mu(x)$ corresponds to an asymmetric confining potential that includes a cubic term in displacement 52] and, consequently, leads to non-linear terms in the equation of motion discussed above.

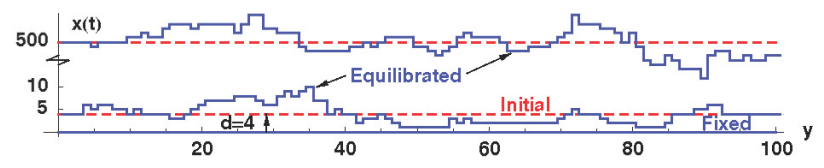

FIG. 9. Snapshot of the MC configuration, $x(y)$ of an isolated step $(\mathrm{d}=500)$ and $\mathrm{d}=4$ for $T=\epsilon / k_{B}$ and $L_{y}=100$. The initial step and the equilibrated step are shown as dashed and solid lines, respectively. The line $x=0$ is the position of the fixed neighboring step. From Ref. 24]. 

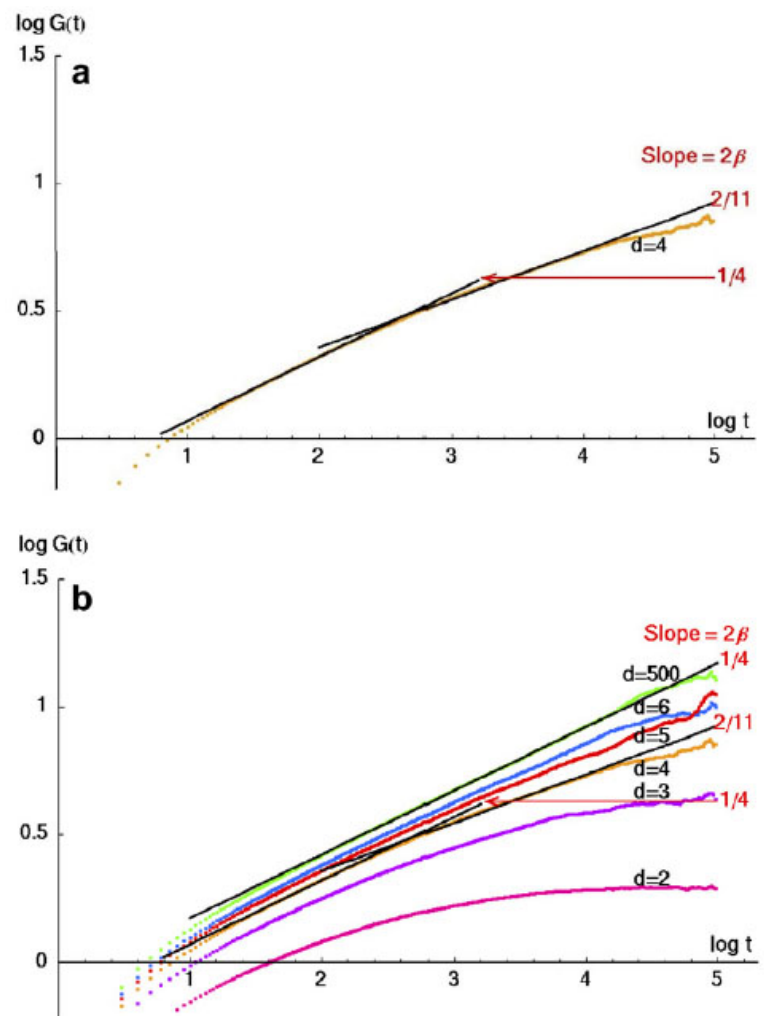

FIG. 10. Log-log plot of $G\left(y_{0}, t\right)$ obtained from $10^{7} \mathrm{MC}$ steps per site after equilibration and averaged over 10 realizations. In panel a) results for $\mathrm{d}=4$ are plotted; the slope is clearly consistent with rather rapid crossover between the predicted values (lines included for convenience) $2 \beta=1 / 4$ at small $t$ and then $2 \beta=2 / 11$. In panel b) are results for values of $d$ between 2 and 500. For $d=2$ logarithmic behavior $(\beta=0)$ is observed, while for large values of $d(d=500) 2 \beta=1 / 4$. Adapted from Refs. 24, 39].

\section{MONTE CARLO SIMULATIONS}

As discussed above, both geometry and interstep interactions break the front-back symmetry of the potential felt by a fluctuating step bordering a facet. Taking both effects into account is not feasible in a simple way. In Section 3 we accounted for the geometry by computing the chemical potential for a closed (circular) step. In this Section we consider a simpler geometry, in which steps are straight, and we consider the effect of the contact repulsion (entropic interaction) between neighboring steps. This will help us to elucidate the role played by step-step interactions in determining the physics of asymmetric confinement in a conserved-volume system. To this end, we assumed SED-limited kinetics, and we carried out standard Monte Carlo (MC) simulations of a "toy" TSK model on a square lattice in which a single "active" step is placed a distance $d$ lattice constants (in the $\hat{x}$ direction) from a second fixed straight step (i.e. a $1+1 \mathrm{D}$ SOS model with this and other restrictions); both steps have projected length $L_{y}[39$. For convenience we

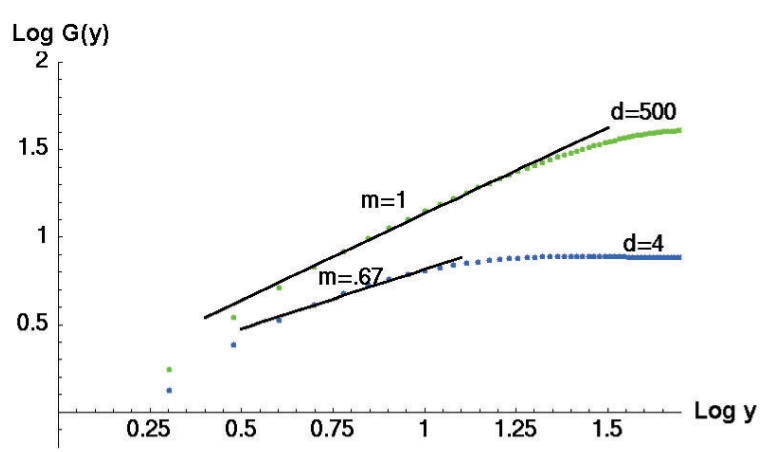

FIG. 11. Log-log plot of $G\left(y, t_{0}\right)$ obtained after equilibration for $d=4$ and $d=500$. The fit of the slope $m$ (i.e. $2 \alpha$ ) - over the linear regime where $y$ is less than the correlation lengthis 0.67 and 1 for $d=4$ and $d=500$, respectively. From Refs. 24, 39].

set $k_{B} T$ to the energy $\epsilon$ of a unit length of step and assume only entropic repulsive interactions between the two steps. The active step evolves by Kawasaki dynamics, with trial moves by "atoms" at the step to neighboring sites along the step. (In SOS language, neighboring sites increase and decrease by one unit.) Most of our runs were done with $L_{y}=100$, with $\sim 10^{8}$ MCS; consistent with the high value of $z$ for SED dynamics, runs with $L_{y}=200$ converge poorly. Figure 9 shows a snapshot of a MC configuration of an essentially isolated step $(d=500[a])$ and for $d=4$, both initially (dashed) and after equilibration (solid) [19, 24].

To simulate confinement, the neighboring step is placed at distances $d=2,3,4,5,6$, and 500. Figure 10 shows $\mathrm{G}(\mathrm{t})$ obtained after equilibration for $10^{7} \mathrm{MCS}$ (MC steps per site) and averaged over 10 realizations. Figure 10a shows results for just $d=4$ for clarity. The fit to the data clearly shows crossover. At early times the slope is $m \sim 0.25$ while later $m \sim 0.18$, close to the predicted values of $2 \beta=1 / 4$ and $2 \beta=2 / 11$ for isolated and facet (confined) step-edge fluctuations, respectively, with SED kinetics. In other words, once the step meanders enough to "become aware" of the fixed step, $G\left(y, t_{0}\right)$ crosses over from random walk behavior to asymmetric conserved-volume confinement, then eventually begins to cross over to the flat late-time behavior of symmetrically confined steps. Figure $10 \mathrm{p}$ adds to Figure $10 \mathrm{a}$ the results for $d=2,3,5,6$, and 500 . For $d=2$ the neighboring step is so close that no simple power-law behavior is evident; passage from early to late behavior is quick, smooth, and broad. As the stepstep distance increases, the time until crossover from $2 \beta=1 / 4$ to $2 \beta=2 / 11$ also increases. When $d>6$, crossover is not seen before the end of the $10^{7} \mathrm{MCS}$, and the slope remains $2 \beta=1 / 4$. Thus, for $d=6$ and especially for $d=500$, much larger than the mean squared width of the step $w^{2}$, the fixed step never significantly influences the active one. That the experimental value of $2 \beta=0.15 \pm 0.03$ is somewhat below $2 / 11$ 
weakly suggests (one- $\sigma$ ) that some physical effect may be acting to reduce the growth exponent. The possibility of extreme damping of fluctuations due to small step spacings, as for $d=2$ in Fig. $10 \mathrm{p}$, is unlikely since the $\mathrm{Pb}$ measurements correspond to $d / w$ values $\sim 5-10$, well above the strong-confinement regime [19]. Thorough analysis of a more detailed model would be needed for quantification.

Figure 11 shows results of $G\left(y, t_{0}\right)$ obtained after equilibration for $d=4$ and $d=500$. The initial linear portion of the log-log-plotted data is fit to a slope $m \sim 0.67$ and $m \sim 1.0$ for $d=4$ and $d=500$, consistent with the prediction of $2 \alpha=2 / 3$ and $2 \alpha=1$ for confined and isolated steps, respectively [24, 39]. However, since the length of the step is limited to 100 and the linear scaling in Fig. 11 is over less than a decade in $y$, these results are not fully conclusive.

As shown in Fig. 10, after random-walk $(2 \beta=1 / 2)$ evolution at the very outset (first few points), $G(t)$ quickly crosses over to isolated-step $(2 \beta=1 / 4)$ behavior. For $d=4$, For $d=2$, confinement is so great that $G(t)$ progresses quickly from initial- to late-time evolution, with no clear intermediate regime.

\section{CONCLUSIONS}

The work presented here is a very good example of the interplay in statistical physics between exact results, scaling arguments, numerical simulations, and experiments. Spohn and coworkers have produced a novel, exact static result. This has motivated us to apply old scaling arguments to derive novel dynamical behaviors. In turn, the latter have opened new avenues for experimentalists to explore. And the results of experiments have motivated numerical simulations of model systems. In particular, for the first time it has been possible to observe experimental evidence for a nonlinear term in equilib- rium fluctuations. The result agrees with our predictions for the case of geometrically confined fluctuations. When power-law temporal correlations are measured, the measured value of the power $\beta$ is significantly smaller than the unconfined exponent of $\beta=1 / 8$, and is within $1 \sigma$ of the predicted value of $\beta=1 / 11$ for a universality class of dynamical scaling with $\alpha=1 / 3$ and $z=11 / 3$. Thanks to the extensions by Spohn and coworkers of earlier links between KPZ behavior and the behavior of facet edges [53, we were able, for the first time (to the best of our knowledge), to provide an example in which a KPZ-type equation of motion accounted for equilibrium fluctuations. We were also able to experimentally verify the predictions of the theory. The experiments spurred a more detailed numerical study of the problem. As a result, it was discovered that the fluctuations and equation of motion of steps at equilibrium are very sensitive to the step environment [54, a discovery that may introduce new opportunities for controlling the fabrication of nanostructures and for understanding new aspects of their dynamic properties.

\section{ACKNOWLEDGMENTS}

Work at University of Maryland has been supported by the UMD-NSF MRSEC under grant DMR 05-20471; TLE is now supported partially by NSF-CHE 07-50334 and 13-05892. Much of this paper is based on extensive collaboration with the experimental surface physics group at UMD, led by Ellen D. Williams until 2010, with ongoing guidance by Janice Reutt-Robey and William G. Cullen, in particular with Masashi Degawa, whose dissertation research accounts for much of the content of this paper. We also benefited from interactions with theory postdoc Ferenc Szalma and students Hailu Gebremarian and Timothy J. Stasevich.
[1] H.-C. Jeong and E.D. Williams, Steps on Surfaces: Experiment and Theory, Surf. Sci. Rep. 34, 175 (1999).

[2] M. Giesen, Step and Island Dynamics at Solid/Vacuum and Solid/Liquid Interfaces, Prog. Surf. Sci. 68, 1 (2001).

[3] I. Lyubinetsky, D.B. Dougherty, T.L. Einstein, and E.D. Williams, Dynamics of Step Fluctuations on a Chemically Heterogeneous Surface of $\mathrm{Al} / \mathrm{Si}(111)-(\sqrt{ } 3 \times \sqrt{ } 3)$, Phys. Rev. B 66, 085327 (2002).

[4] O. Bondarchuk, D.B. Dougherty, M. Degawa, E.D. Williams, M. Constantin, C. Dasgupta and S. Das Sarma, Correlation Time for Step Structural Fluctuations, Phys. Rev. B 71, 045426 (2005).

[5] L. Kuipers, M. S. Hoogeman, J. W. M. Frenken, and H. van Beijeren, Step and kink dynamics on $\mathrm{Au}(110)$ and $\mathrm{Pb}$ (111) studied with a high-speed STM, Phys. Rev. B 52, 11387 (1995); S. Speller, W. Heiland, A. Biedermann, E. Platzgummer, C. Nagl, M. Schmid, and P. Varga, An STM study of the step structure of $\mathrm{Pb}(110)$ and $\mathrm{Pb}(111)$,
Surf. Sci. 333, 1056 (1995).

[6] P. C. Hohenberg and B. I. Halperin, Theory of dynamic critical phenomena, Rev. Mod. Phys. 49, 435 (1977).

[7] T.L. Einstein and S.V. Khare, Step Fluctuations: Fruom Equilibrium Analysis to Step Unbunching and Cluster Diffusion in a Unified Picture, in P.M. Duxbury and T.J. Pence, editors, Dynamics of Crystal Surfaces and Interfaces (Plenum, New York, 1997), 83.

[8] N. C. Bartelt, T.L. Einstein, and E. D. Williams, Measuring Surface Mass Diffusion Coefficients by Observing Step Fluctuations, Surface Sci. 312, 411 (1994).

[9] N. C. Bartelt, J.L. Goldberg, T.L. Einstein, E. D. Williams, J.C. Heyraud, and J.J. Métois, The Brownian Motion of Steps on $\operatorname{Si}(111)$, Phys. Rev. B 48, 15453 (1993).

[10] W. Selke and M. Bisani, Diffusive and subdiffusive step dynamics, in: Anomalous Diffusion: From Basics to Applications (Lecture Notes in Physics 519, Springer); 
eds.: R. Kutner, A. Pekalski, and K. Sznajd-Weron, 298 (1999).

[11] Z. Kuntová, Z. Chvoj, V. Šíma, and M. C. Tringides, Limitations of the thermodynamic Gibbs-Thompson analysis of nanoisland decay, Phys. Rev. B 71, 125415 (2005).

[12] M. Degawa, F. Szalma and E.D. Williams, Nanoscale Equilibrium Crystal Shape, Surf. Sci. 583, 126 (2005).

[13] Hailu Gebremariam Bantu, Terrace Width Distribution and First Passage Probabilities for Interacting Steps, Ph.D. thesis, U. of Maryland (2005).

[14] P.L. Ferrari, M. Prähofer, and H. Spohn, Fluctuations of an atomic ledge bordering a crystalline facet, Phys. Rev. E 69, 035102(R) (2004).

[15] P.L. Ferrari and H. Spohn, Step Fluctuations for a Faceted Crystal, J. Stat. Phys. 113, 1 (2003).

[16] Patrik L. Ferrari, Shape fluctuations of crystal facets and surface growth in one dimension, Ph.D. Thesis, TU München (2004), available at http://tumb1.ub.tum.de/publ/diss/ma/2004/ferrari.html

[17] H. Spohn, Exact solutions for KPZ-type growth processes, random matrices, and equilibrium shapes of crystals, Physica A 369, 71 (2006).

[18] A. Pimpinelli, J. Villain, D.E. Wolf, J.J. Métois, J.C. Heyraud, I. Elkinani and G. Uimin, Equilibrium step dynamics on vicinal surfaces, Surf. Sci. 295, 143 (1993).

[19] M. Degawa, T.J. Stasevich, W.G. Cullen, A. Pimpinelli, T.L. Einstein, and E.D. Williams, Distinctive Fluctuations in a Confined Geometry, Phys. Rev. Lett. 97, 080601 (2006).

[20] A. Pimpinelli, M. Degawa, T.L. Einstein, and E.D. Williams, A Facet Is Not an Island: Step-Step Interactions and the Fluctuations of the Boundary of a Crystal Facet, Surf. Sci. Lett. 598, L355 (2005).

[21] T.L. Einstein, Alberto Pimpinelli, M. Degawa, T.J. Stasevich, W.G. Cullen, and E.D. Williams, 95th Statistical Mechanics Conference, Rutgers, Piscataway, May 2006.

[22] M. Bisani and W. Selke, Step fluctuations and random walks, Surf. Sci. 437, 137 (1999).

[23] N. C. Bartelt, T. L. Einstein, and E. D. Williams, The Role of Step Collisions on Diffraction from Vicinal Surfaces, Surf. Sci. 276, 308 (1992).

[24] M. Degawa, T.J. Stasevich, A. Pimpinelli, T.L. Einstein, and E.D. Williams, Facet-edge Fluctuations with Periphery Diffusion Kinetics, Surf. Sci. 601, 3979 (2007).

[25] K. Thürmer, J.E. Reutt-Robey, and E.D. Williams, Nucleation limited crystal shape transformations, Surf. Sci. 537, 123 (2003).

[26] Physically, steps - in contrast to fermions - actually can touch, just not cross, leading to a finite-size correction to the standard fermion results. See R. Sathiyanarayanan, A. BH. Hamouda, and T.L. Einstein, Terrace-width Distributions of Touching Steps: Modification of the Fermion Analogy, with Implications for Measuring Stepstep Interactions, Phys. Rev. B 80, 153415 (2009).

[27] In Ref. [14] $A$ is defined as $b_{\infty}^{\prime \prime}$; writing $\mathcal{A}=(1 / 2) A$ simplifies Eq. (6).

[28] E.E. Gruber and W.W. Mullins, On the Theory of Anisotropy of Crystalline Surface Tension, J. Phys. Chem. Solids 28, 6549 (1967); V. L. Pokrovsky and A. L. Talapov, Ground State, Spectrum, and Phase Diagram of Two-Dimensional Incommensurate Crystals, Phys. Rev. Lett. 42, 65 (1979).
[29] P.M. Duxbury and T.J. Pence, editors, Dynamics of Crystal Surfaces and Interfaces (Plenum, New York, 1997): proceedings of a workshop in Traverse City in August 1996, at which the term was coined and then used by several speakers.

[30] $\vartheta=\theta-\arctan \left[r_{\theta}(\theta) / r(\theta)\right]$, as derived in S. V. Khare, S. Kodambaka, D. D. Johnson, I. Petrov, and J. E. Greene, Determining absolute orientation-dependent step energies: a general theory for the Wulff-construction and for anisotropic two-dimensional island shape fluctuations, Surf. Sci. 522, 75 (2003); S. Kodambaka, S. V. Khare, V. Petrova, D. D. Johnson, I. Petrov, and J. E. Greene, Absolute orientation-dependent anisotropic TiN(111) island step energies and stiffnesses from shape fluctuation analyses, Phys. Rev. B 67, 035409 (2003).

[31] S. V. Khare, N. C. Bartelt, and T. L. Einstein, Diffusion of Monolayer Adatom and Vacancy Clusters: Langevin Analysis and Monte Carlo Simulations of Their Brownian Motion, Phys. Rev. Lett. 75, 2148 (1995); Brownian Motion and Shape Fluctuations of Single Layer Adatom and Vacancy Clusters on Surfaces: Theory and Simulations, Phys. Rev. B 54, 11752 (1996).

[32] A. Pimpinelli and J. Villain, Physics of Crystal Growth (Cambridge University Press, Cambridge, 1998).

[33] Note $\left\langle\eta_{C}(\theta, t) \eta_{C}\left(\theta^{\prime}, t^{\prime}\right)\right\rangle \propto-\rho_{0}^{-2} \partial^{2} \delta\left(\theta-\theta^{\prime}\right) / \partial \theta^{2} \delta\left(t-t^{\prime}\right)$, while $\left\langle\eta(\theta, t) \eta\left(\theta^{\prime}, t^{\prime}\right)\right\rangle \propto \delta\left(\theta-\theta^{\prime}\right) \delta\left(t-t^{\prime}\right)$.

[34] M. Kardar, G. Parisi, and Y. C. Zhang, Dynamic Scaling of Growing Interfaces, Phys. Rev. Lett. 56, 889 (1986).

[35] T. Sun, H. Guo and M. Grant, Dynamics of driven interfaces with a conservation law, Phys. Rev. A 40, 6763 (1989).

[36] A.-L. Barabási and H. E. Stanley, Fractal Concepts in Surface Growth (Cambridge U. Press, Cambridge, 1995).

[37] B. Krishnamachari, J. McLean, B. Cooper, and J. Sethna, Gibbs-Thomson formula for small island sizes: Corrections for high vapor densities, Phys. Rev. B 54, 8899 (1996).

[38] M. Degawa and E.D. Williams, Barriers to shape evolution of supported nano-crystallites, Surf. Sci. 595, 87 (2005).

[39] M. Degawa, Equilibrium and Non-Equilibrium Properties of Finite-Volume Crystallites, Ph.D. thesis, University of Maryland (2006); also Ref. [19.

[40] H. G. E. Hentschel and F. Family, Scaling in open dissipative systems, Phys. Rev. Lett. 66, 1982 (1991).

[41] Y. Kim, S. Y. Yoon, and H. Park, Fluctuations of selfflattening surfaces, Phys. Rev. E 66, 040602(R) (2002) present MC results for a restricted solid-on-solid (RSOS) model, which also includes a somewhat artificial mechanism to limit the fluctuation width. They find $\beta \simeq 2 / 9$ and $z \simeq 3 / 2$.

[42] M. Uwaha and P. Nozières, Crystal Shapes Viewed as Mechanical Equilibrium of Steps, in Morphology and Growth Unit of Crystals, edited by I. Sunagawa (Terra Scientific Publishing, Tokyo, 1989), 17.

[43] W. L. Winterbottom, Equilibrium Shape of a Small Particle in Contact with a Foreign Substrate, Acta Metall. Mater. 15, 303 (1967); P. Müller and R. Kern, Equilibrium nano-shape changes induced by epitaxial stress (generalised Wulf-Kaishew theorem), Surf. Sci. 457, 229 (2000).

[44] M. Wortis, Equilibrium Crystal Shapes and Interfacial Phase Transitions, in Chemistry and Physics of Solid Surfaces VII, edited by R. Vanselow and R. F. Howe 
(Springer, Berlin, 1988), 367; P. Nozières, Shape and Growth of Crystals, in Solids far from Equilibrium, edited by C. Godrèche (Cambridge U. Press, Cambridge, 1992), 1; S. Balibar, H. Alles, and A.Ya. Parshin, The surface of helium crystals, Rev. Mod. Phys. 77, 317 (2005); A. Pavlovska et al., Surf. Sci. 326, 101 (1995); C. Rottman, M. Wortis, J.C. Heyraud, and J.J. Métois, Equilibrium Shapes of Small Lead Crystals: Observation of Pokrovsky-Talapov Critical Behavior, Phys. Rev. Lett. 52, 1009 (1984).

[45] K. Arenhold, S. Surnev, H.P. Bonzel, and P. Wynblatt, Step energetics of $\mathrm{Pb}(111)$ vicinal surfaces from facet shape, Surf. Sci. 424, 271 (1999); C. Bombis, A. Emundts, M. Nowicki, and H.P. Bonzel, Absolute surface free energies of Pb, Surf. Sci. 511, 83 (2002); M. Nowicki et al., New J. Phys. 4, 60 (2002).

[46] K. Thürmer, J.E. Reutt-Robey, E.D. Williams, M. Uwaha, A. Emundts, and H.P. Bonzel, Step Dynamics in 3D Crystal Shape Relaxation, Phys. Rev. Lett. 87, 186102 (2001).

[47] N. Akutsu and Y. Akutsu, Statistical mechanical calculation of anisotropic step stiffness of a two-dimensional hexagonal lattice-gas model with next-nearest-neighbour interactions: application to $\mathrm{Si}(111)$ surface, J. Phys.Cond. Mat. 11, 6635 (1999); M. Nowicki, C. Bombis, A. Emundts, and H. P. Bonzel, Absolute step and kink formation energies of $\mathrm{Pb}$ derived from step roughening of two-dimensional islands and facets, Phys. Rev. B 67, 075405 (2003).

[48] Direct experimental observation, e.g. of the spatial correlation function on a quenched crystallite, would be needed to obtain $\alpha$ for facet-edge fluctuations.
[49] T. Ihle, C. Misbah, and O. Pierre-Louis, Equilibrium step dynamics on vicinal surfaces revisited, Phys. Rev. B 58, 2289 (1998); S.V. Khare and T.L. Einstein, Unified view of step-edge kinetics and fluctuations, Phys. Rev. B 57, 4782 (1998); M. Ondrejcek, M. Rajappan, W. Swiech, and C. P. Flynn, Step fluctuation studies of surface diffusion and step stiffness for the $\mathrm{Ni}(111)$ surface, Phys. Rev. B 73, 035418 (2006).

[50] E. Le Goff, L. Barbier, and B. Salanon, Timespace height correlations of thermally fluctuating 2-d systems. Application to vicinal surfaces and analysis of STM images of Cu(1 1 5), Surf. Sci. 531, 337 (2003); M. Ondrejcek, W. Swiech, G. Yang, and C.P. Flynn, Crossover from bulk to surface diffusion in the fluctuations of step edges on Pt(111), Phil. Mag. Lett. 84, 69, 417 (2004); M. Ondrejcek, W. Swiech, M. Rajappan, and C. P. Flynn, Fluctuation spectroscopy of step edges on $\mathrm{Pt}(111)$ and Pd(111), Phys. Rev. B 72, 085422 (2005).

[51] N. C. Bartelt, T.L. Einstein, and E. D. Williams, The Influence of Step-Step Interactions on Step Wandering, Surf. Sci. Lett. 240, L591 (1990).

[52] T. J. Stasevich, Hailu Gebremariam, T.L. Einstein, M. Giesen, C. Steimer, and H. Ibach, Low-Temperature Orientation Dependence of Step Stiffness on 111 Surfaces, Phys. Rev. B 71, 245414 (2005).

[53] J. D. Shore and D. J. Bukman, Coexistence point in the six-vertex model and the crystal shape of fcc materials, Phys. Rev. Lett. 72, 604 (1994); J. Neergaard and M. den Nijs, Crossover Scaling Functions in One Dimensional Dynamic Growth Models, Phys. Rev. Lett. 74, 730 (1995).

[54] C. Tao, T. J. Stasevich, T.L. Einstein, and E. D. Williams, Step Fluctuations on $\mathrm{Ag}(111)$ Surfaces with C60, Phys. Rev. B 73, 125436 (2006). 